\title{
Identifiability Analysis of a Pressure-Depending Alveolar Recruitment Model
}

\author{
Christoph Schranz****, Paul D. Docherty**, Yeong Shiong Chiew**, Knut Möller*, J. Geoffrey Chase** \\ *Institute for Technical Medicine, Furtwangen University, \\ Villingen-Schwenningen, Germany, (e-mail: scc@hs-furtwangen.de) \\ **Department of Mechanical Engineering, University of Canterbury \\ Christchurch, New Zealand
}

\begin{abstract}
Patient-specific physiological models of respiratory mechanics can offer insight into patient state and pulmonary dynamics that are not directly measurable. Thus, significant potential exists to evaluate and guide patient-specific lung protective ventilator strategies for Acute Respiratory Distress Syndrome (ARDS) patients. To assure bedside-applicability, the physiological model must be computationally efficient and identifiable from the limited available data, while also capturing dominant dynamics and trends observed in ARDS patients. In this work, an existing static recruitment model is enhanced by considering alveolar distension and implemented in a novel time-continuous dynamic respiratory mechanics model. A hierarchical gradient descent approach is used to fit the model to lowflow test responses of 12 ARDS patients. Identified parameter values were physiologically plausible and capable of reproducing the measured pressure responses with very high accuracy (Overall median percentage fitting error: MPE $=1.84 \%$ [IQR: $1.77 \%$ to $2.18 \%]$ ). Structural identifiability of the model is proven, but a practical identifiability analysis of the results shows a lack of convexity on the error-surface for some patients due to reduced information content within the measured data set. Overall, the model presented is physiologically and clinically relevant, captures ARDS dynamics, and uses clinically descriptive parameters. The patient-specific models show their ability to capture pulmonary dynamics directly relevant to patient condition and clinical guidance. These characteristics cannot be directly measured without such a validated model.
\end{abstract}

Keywords: Respiratory Mechanics, Alveolar Recruitment, Parameter Identification, Identifiability

\section{INTRODUCTION}

Mathematical models of respiratory mechanics can be used to predict various ventilator settings and could support the evaluation of patient-specific lung protective lung ventilator settings in case of Acute Respiratory Distress Syndrome (ARDS) (Lozano et al., 2008, Sundaresan et al., 2011). Optimal predictions in real-time at the bedside require computationally efficient models that are identifiable with the limited available information of measured flow rate and airway pressure. Hence, the model must be as simple as possible while capturing all relevant dynamics. In case of ARDS, dominant dynamics include primary alveolar recruitment increasing lung compliance by opening up new alveolar units (Gattinoni et al., 2006). Furthermore, alveolar distension effects at higher pressure levels which indicate over-inflation due to stiffening of the lung tissue must be known to define optimal tidal volume. No practical methods are currently available to capture these effects and thus guide therapy.

Hickling's model formulation of ARDS lungs (Hickling, 1998) is an established principle to produce pressure-volume characteristics of ARDS patients. The model is currently applied successfully in various forms: (Markhorst et al., 2004) performed simulations to predict optimal lung protective airway pressures. (Sundaresan et al., 2009) used a modified version of Hickling's model to estimate opening pressures based on patient data in order to calculate optimal levels of Positive End-Expiratory Pressure (PEEP).

This paper introduces the implementation of Hickling's recruitment principle in a time-continuous model to predict pressure-responses of ARDS patients. The model is individualized with clinical data and its identifiability is critically checked.

\section{MATERIALS \& METHODS}

\subsection{Models}

The introduced models are applied according to volumecontrolled ventilation with the flow rate $(\dot{V}=d V / d t)[\mathrm{mL} / \mathrm{s}]$ as model input and airway pressure $\left(p_{a w}\right)$ [mbar] as model output. Alveolar pressure $\left(p_{a}\right)$ [mbar] is an unmeasured intermediate variable.

$1^{\text {st }}$ Order Model of Respiratory Mechanics (FOM): The linear FOM consists of a serial arrangement of a resistance $R_{F O M}$ (mbar·s $/ \mathrm{mL}$ ) and a compliance $C_{F O M}(\mathrm{~mL} / \mathrm{mbar})$.

$p_{a w}=R_{F O M} \dot{V}+p_{a}$

$\dot{p}_{a}=C_{F O M}{ }^{-1} \dot{V}$ 
$R_{F O M}$ represents the total airway resistance and resistive tissue contributions, while $C_{F O M}$ is a measure for the elasticity of the respiratory system (lung and chest wall).

Pressure Dependent Recruitment Model (PRM): The pressure-dependent recruitment model (PRM) implements a pressure-dependent alveolar recruitment principle (Hickling, 1998), and considers alveolar distension effects (Salazar and Knowles, 1964) implemented in the FOM.

Hickling's recruitment principle divides the lung into 30 horizontal layers to capture different levels of superimposed pressure from $S P_{n}=0$ to $14.5 \mathrm{mbar}$ in $0.5 \mathrm{mbar}$ increments. Each layer represents a set of alveolar units that are either recruited $\left(H_{n}=1\right)$ or not recruited $\left(H_{n}=0\right)$. The Treshold Opening Pressure $(T O P)$ is a patient-specific pressure that needs to be exceeded in addition to the $S P_{n}$ to recruit and stabilize alveolar units within a layer $n$.

$H_{n}= \begin{cases}0, & p_{a} \leq S P_{n}+T O P \\ 1, & p_{a}>S P_{n}+T O P\end{cases}$

with $n=0,1,2, \ldots 30$

Once a layer is recruited, the layer compliance contributes to the overall lung compliance. The initial compliance of a recruited layer is defined $C_{L}$. At the beginning of inspiration, a certain amount of alveolar units are open, defining the functional residual volume. The overall initial compliance of these alveoli is denoted as $C_{F R C}$. The compliances of the initially opened layer and the recruited layer decrease exponentially according to the distension model (Salazar and Knowles, 1964). The exponential decrease is described by parameter $K$. The resulting model of a pressure depending compliance $C_{P R M}\left(p_{a}\right)$ is embedded into the structure of the FOM (Eq. 1a-b), replacing the static compliance $C_{F O M}$ yielding the PRM:

$p_{a w}=R_{P R M} \dot{V}+p_{a}$

$\dot{p}_{a}=\left[C_{F R C} e^{-K p_{a}}+C_{L} \sum_{n=1}^{30} H_{n} e^{-K\left(p_{a}-T O P-S P_{n}\right)}\right]^{-1} \dot{V}$

The PRM patient-specific parameter set is defined:

$$
X=\left\{R_{P R M}, C_{P R M}, \Theta, K, T O P\right\}
$$

where $R_{P R M}$ represents the total airway resistance and $C_{P R M}$ captures the overall maximal compliance of the completely recruited lung, without considering distension effects:

$C_{P R M}=C_{F R C}+30 C_{L}$

$\Theta$ is the ratio of opened alveolar units before inspiration compared to the overall amount of total units. Thus, $\Theta$ takes a value from 1.0, for an initially completely recruited lung, to a value of 0.0 for a completely collapsed lung.

$\Theta=\frac{C_{F R C}}{C_{P R M}}$

\subsection{Data}

Measurements of twelve mechanically ventilated (MV) patients were selected from a previous ARDS - Study, where Low-Flow (LF) manoeuvres were performed using an
Evita4Lab-System (Stahl et al., 2006). The measurements consisted of flow rate and airway pressure signals sampled at $125 \mathrm{~Hz}$. The study was approved by the local ethics committees of the participating university hospitals. Informed consent was signed by patients or their legally authorized representative. Please refer to (Stahl et al., 2006) for a detailed description of the experimental setup. During the LF manoeuvre the lung is inflated by an extremely low constant gas flow of $33 \mathrm{~mL} / \mathrm{s}$ until the airway opening pressure reaches $45 \mathrm{mbar}$, enabling a quasi-static pressure/volume relationship (Kretschmer et al., 2010).

\subsection{Parameter Identification}

Patient specific parameter values were defined by fitting modelled responses to the experimental data. The best fit was obtained by minimizing the sum of squared error (SSE) between measured and simulated $p_{a w}$.

$$
S S E=\sum\left(p_{a w, \text { meas }}-p_{a w}\right)^{2}
$$

Parameter identification of the PRM leads to a non-linear problem and is thus performed by gradient-based algorithms.

The computational intensity of gradient-based parameter identification rises with the increasing numbers of parameters, as a range of various parameter constellations or local minima appear as possible solutions. Accurate initial parameter values can significantly reduce the incidence of finding local minima. Thus, a hierarchical parameter identification process is applied (Schranz et al., 2011).

The hierarchical method provides patient-specific initial values by identifying simpler models with fewer variable parameters first (Schranz et al., 2011). These first results provide appropriate initial values for the identification of the next, more complex model.

In the given case of PRM identification, the linear FOM can be seen as a reduced model of the PRM with setting $\Theta=1$ and $K=0$. Thus, a-priori identification of the FOM provides convenient initial values for $R_{P R M}$ and $C_{P R M}$ for the subsequent PRM identification. The PRM parameters were identified with the following steps:

1. Identify the FOM (Eq. 1a-b) with Multiple Linear Regression, using $\dot{V}$ as model input and $p_{a w}$ as model output. This yields parameters $R_{F O M}$ and $C_{F O M}$.

2. Simulate the FOM (Eq. 1a-b) with given $\dot{V}$ as model input to evaluate the maximal alveolar pressure $p_{a, \max }$.

3. Set the initial values for the patient-specific parameter set $\mathbf{X}$ (Eq. 4) for subsequent gradient-based PRM parameter identification according to Table 1.

4. Calculate the initial model parameter values $C_{F R C}$ and $C_{L}$ for PRM simulation and parameter identification according to Eq. 5, 6 .

5. PRM parameter identification is undertaken using a Trust-Region Algorithm with $\dot{V}$ as model input and $p_{a w}$ as model output. This yields patient-specific model parameters $R_{P R M}, C_{F R C}, C_{L}, K, T O P$.

6. Calculate $C_{P R M}$ and $\Theta$ according to Eq. 5, 6 to fulfil the identified set $\mathbf{X}$. 
Table 1. Setting convenient initial values for the patientspecific parameter set $\boldsymbol{X}$ for gradient-based PRM identification

\begin{tabular}{|c|c|}
\hline Parameter & Comment \\
\hline$R_{P R M}=R_{F O M}$ & hierarchically derived \\
\hline$\Theta=0.5$ & $\begin{array}{l}\text { according to general findings in CT- } \\
\text { Images of ARDS patients (Albaiceta } \\
\text { et al., 2004) }\end{array}$ \\
\hline$K=0.0311 / \mathrm{mbar}$ & arbitrarily chosen \\
\hline$C_{P R M}=\frac{K C_{F O M} p_{a, \max }}{1-e^{-K p_{a, \max }}}$ & $\begin{array}{l}\text { hierarchically derived (Schranz et al., } \\
\text { 2012) }\end{array}$ \\
\hline$T O P=0,2,4, \ldots 12$ & $\begin{array}{l}\text { Identify with given range. The } \\
\text { solution with the lowest SSE is } \\
\text { selected }\end{array}$ \\
\hline
\end{tabular}

\subsection{Identifiability Analysis}

Structural Identifiability: A necessary model prerequisite for successful parameter identification are mathematically distinct model parameters (Docherty et al., 2011). Therefore the model should be checked for a-priori structural identifiability, assuring that the model parameters of the model can be uniquely regained, given ideal noise-free measurements of input and output, and an error-free model structure. The PRM was tested for structural identifiabilty using DAISY (Bellu et al., 2007).

However, even if structural identifiability is proven, the model may still be non-identifiable (Bellu et al., 2007). Furthermore, structural identifiability also does not prevent error-mapping methods from being caught in local minima. Thus, conclusive model evaluation must be undertaken with clinically realistic conditions.

Practical Identifiability: Practical non-identifiability is observed if the SSE around the global minimum remains below a certain threshold. In these regions, changes in SSE are negligible, despite significant variation in model parameter values (Raue et al., 2009). Practical identifiability analysis was assessed, using error-change-rate.

After parameter identification, the error-change-rate $(E)$ around any reported minima was calculated by allowing a single parameter shifting up to $\pm 10 \%$ from the located minima. The SSE was computed and the error-change-rate was plotted as a function of a change in a single model parameter featuring a $0.4 \%$ resolution.

\section{RESULTS}

According to DAISY, the PRM parameters are mathematically distinct and thus the model has global structural identifiability

The cohort statistics $(\mathrm{N}=12)$ and results of Patients 1,5 and 11 as examples are shown in Table 2, with corresponding median percentage error (MPE) of the model fit. The measured and modeled pressure responses of Patient 1, 5 and 11 are shown in Fig. 1. The error-change-rates of all patients around the reported minima are shown in Fig. 2.
Table 2. Patient-specific model parameters and cohort statistics $(\mathbf{N}=12)$ of identified PRM model parameters

\begin{tabular}{rrrccrc}
\hline $\begin{array}{c}\text { Pat. } \\
\text { Nr. }\end{array}$ & $\begin{array}{c}R_{P R M} \\
(\mathrm{mbar} \cdot \mathrm{s} / \mathrm{mL})\end{array}$ & $\begin{array}{c}C_{P R M} \\
(\mathrm{~mL} / \mathrm{mbar})\end{array}$ & $\begin{array}{c}\Theta \\
(1)\end{array}$ & $\begin{array}{c}K \\
(1 / \mathrm{mbar})\end{array}$ & $\begin{array}{c}T O P \\
(\mathrm{mbar})\end{array}$ & $\begin{array}{c}M P E^{I} \\
(\%)\end{array}$ \\
\hline 1 & 0.093 & 96.18 & 0.48 & 0.035 & 4.9 & 0.77 \\
5 & 0.131 & 42.97 & 0.67 & 0.000 & 1.0 & 1.41 \\
11 & 0.012 & 50.41 & 0.46 & 0.031 & 0.0 & 2.58 \\
\hline Min & 0.012 & 33.90 & 0.24 & 0.000 & 0.0 & 1.21 \\
$\mathrm{Q}_{25}$ & 0.039 & 48.11 & 0.41 & 0.009 & 1.5 & 1.77 \\
Med & 0.063 & 74.56 & 0.47 & 0.019 & 5.7 & 1.84 \\
$\mathrm{Q}_{75}$ & 0.112 & 112.32 & 0.53 & 0.033 & 10.1 & 2.18 \\
Max & 0.199 & 132.56 & 0.67 & 0.056 & 11.0 & 4.07 \\
\hline
\end{tabular}

${ }^{1}$ Median Percentage Error (MPE) of the model fit

The reported parameter values are within physiologically plausible ranges and the PRM reproduces the measured pressure curves with high accuracy and minimal errors.

In general, the error-change-rate in Fig. 2 is most sensitive to variance in $C$, as it produces the highest rate of change across all variables. In contrast, the error surfaces in $R$ and TOP and partially in $K$ are comparatively flat.

The pressure response of Patient 1 in Fig. 1 shows distinct indications for recruitment and distension effects, visible by a curved pressure response. The parameter specific errorchange-rates of this patient are comparatively sensitive allowing successful parameter identification.

Measured data of Patient 5 shows a quasi-linear pressure increase and no evidence of recruitment or distension effects. The identified parameter $K$ was found to be 0.000 (Table 2) and the error-change-rate of Patient 5 in Fig. 2 are flat with respect to $K$ and $T O P$.

Patient 11 shows distinct distension effects but limited resistive pressure steps at the beginning of inspiration. These effects can be related to a flat error-change-rate in terms of $R$ and TOP in Figure 2, whereas the error-dependency with respect to parameter $K$ is comparatively sensitive.

\section{DISCUSSION}

The proposed PRM fits the measured data with high accuracy indicated by low residuals. The model appears able to capture the observed dynamics of ARDS patients with resulting parameter values within physiological ranges for cases with relevant dynamics in the measured response.

The physical principles captured in the PRM represent respiratory dynamics at alveolar scale. The model features recruitment effects, as well as alveolar distension, and allows continuous simulations of respiratory mechanics with respect to time. Each model parameter is descriptive and directly physiologically relevant. $R$ and $C$ offer clinically important insights into the overall airway resistance and lung stiffness. Parameter $K$ is a measurement of how fast a lung unit reaches over-inflation with respect to increasing pressure. Thus, a relatively high value of $K$ would mean that over distension can be reached even in low pressure regions. $\Theta$ and TOP describe the alveolar recruitability. $\Theta$ offers estimates on the fraction of initially recruited alveoli within the lung 

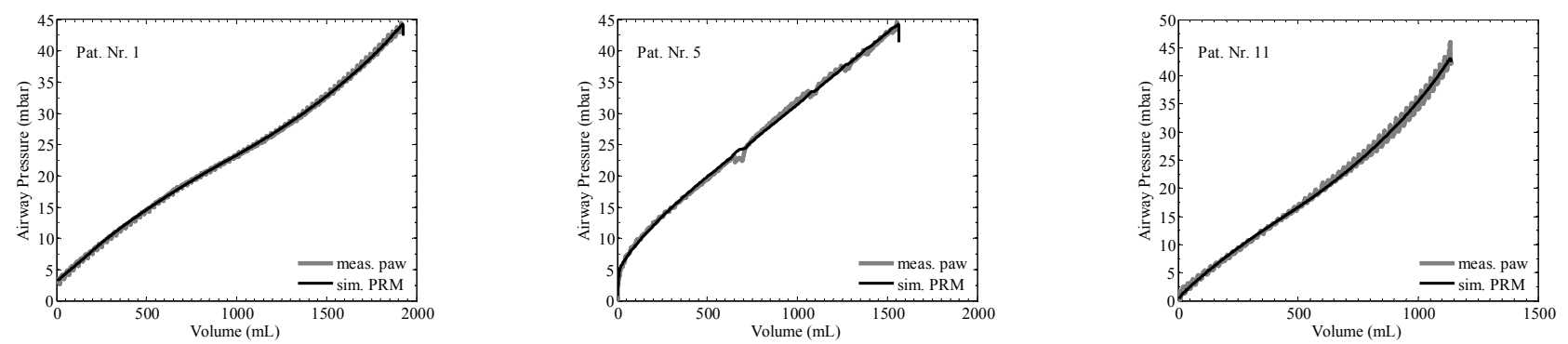

Fig. 1. Measured and simulated pressure responses of patient-specific PRM utilizing the measured flow rate as model input.
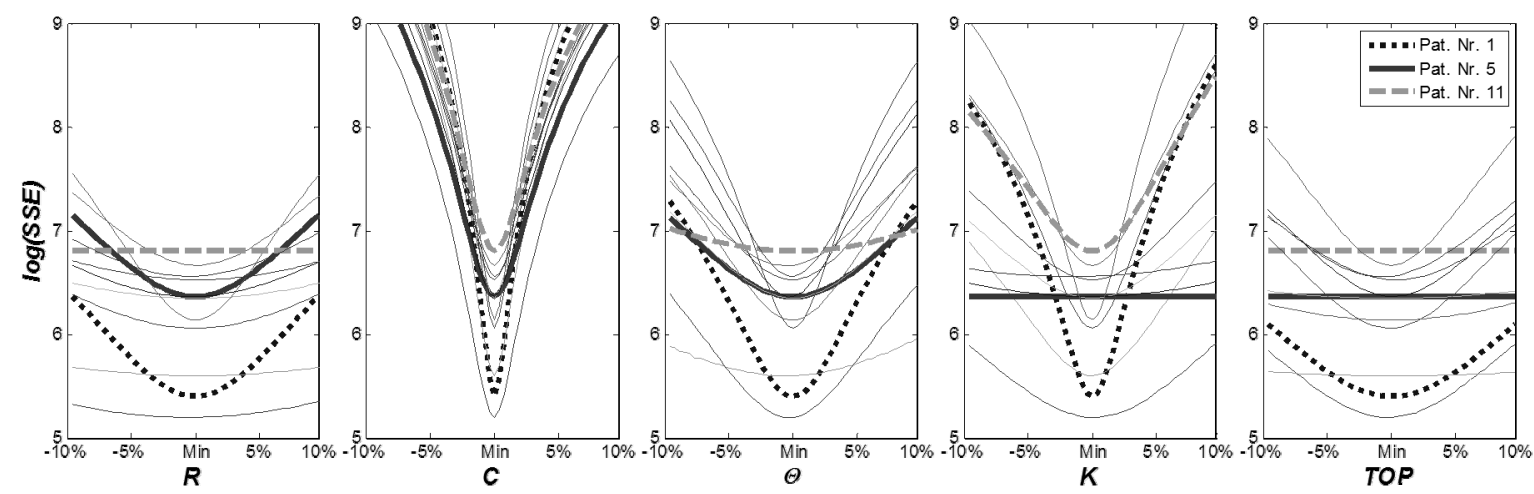

Fig. 2. Error gradients in the vicinity of the identified parameter values of the entire cohort.

compared to the total number of alveoli and TOP seems to be highly relevant in terms of recruitability to guide clinical decision making (Sundaresan et al., 2011).

Practical PRM identifiability seems to be critical in patients, that did not experience an upwards convexity in the pressure response or indicate a quasi-linear pressure increase during the experimental protocol. In cases of limited distension effects, the reported values for $K$ approach 0.000 leading to practical non-identifiability due to missing information content within the data. These cases are indicated by the near flat-lines in Figure 2.

Likewise, in patients with quasi-linear pressure responses, the typical ARDS characteristics are not present. In particular, when recruitment effects (that are typical of most ARDS patient responses) are not observable in the measured data the identifiability of opening pressures and distension parameters is impaired.

The lack of information in the data is indicated in the errorchange-rates in Fig. 2 by relatively wide flat trends for some patients. Gradient-based algorithms occasionally terminate as soon as these flat regions are approached, leading to parameter values relatively far from a true minimum. These wide flat trends are mainly observed in terms of the variables $R, T O P$ and for some patients in $K$. These outcomes emphasise the difficulties parameter identification of this specific model when the characteristics the model is designed to capture are not present in the data set. Alternatively, this analysis shows also which features must be present in the data to allow successful PRM identification. Therefore, successful parameter identification of the PRM requires a curved pressure response indicating recruitment effects and upwards convexity as some evidence of over-distension.

To ensure that the dataset has sufficient information to reliably identify $K$, the tidal volume could potentially be increased to cause some evidence of over-inflation and a resulting distinct upwards convexity in the pressure response. Similarly, a pronounced pressure-step at the beginning of inflation is required to support successful resistance estimation. If one of these features is not present, the model should be reduced or population values could be applied for the particular parameter.

In cases where practical identification of $K$ is inhibited by a lack of distension in the measured data, it may be appropriate to use a population value of $K$. The assumption of a global population value for $K$ may remove the need for distension to be reached during clinical protocols. In cases with significant distension, enforcing a population value for $K$ may cause a poor model fit-to-data and may modulate other model parameters away from appropriate values.

Similarly, it may be reasonable to assume that a resistive influence might be negligible during the given low-flow experiments. Therefore, a model reduction might be practical in the given experiment.

While, the PRM proved to be structural identifiable using DAISY, it can be shown that practical identifiability of the proposed model was not assured with the available airway pressure and flow rate data. Therefore, further investigation is necessary to verify whether the model should be reformulated to simplify parameter identification, particularly in cases wherein distension is not encountered in the experimental data. As practical identifiability is closely 
related to the quality of underlying data, certain preconditions could be elaborated to define a suitable experiment to collect data which allow successful identification.

In the cases wherein the dataset allowed robust identification of the PRM parameters, the model successfully captured the respiratory pressure-volume kinetics by modelling mechanics down in the alveolar scale. Hence, physiological insight gained from the model could potentially be used to optimise ventilator therapy. However, in cases where the dataset did not contain sufficient information for robust model identification, the identified PRM parameters values were of limited value.

However, the physiological interpretations of the model parameters are only valid if the model assumptions are correct. Although, the true recruitment mechanisms are still unknown, several clinical studies (Crotti et al., 2001, Schiller et al., 2003, Pulletz et al., 2011) support the recruitment principle according to Hickling's definition. Thus, the clinical relevance of the PRM has yet to be validated.

In datasets where the PRM was practical identifiable, the model will be more universally useful in a clinical environment, as it offers highly accurate model simulations and physiologically meaningful patient-specific parameters. The PRM model requires only measurements of airway pressure and flow rates that are readily available and noninvasively obtained. The information gained could be clinically and physiologically relevant in the evaluation of lung protective settings and strategies. The model also provides time-continuous simulations of various flow rates. Therefore, it has added potential to be implemented as an additional submodel, interacting with additional physiological models in dynamically generated models for medical decision support for mechanical ventilation (Kretschmer et al., 2011, Sundaresan and Chase, 2011).

\section{CONCLUSION}

A direct, physiologically relevant model was proposed that is able to fit the observed dynamics of ARDS patients with high accuracy. The model parameters are descriptive, clinically relevant and show significant potential to provide unique insight to guide therapy and support lung protective ventilation strategies. Its structural identifiability is proven assuring successful parameter identification under noise-free data and error-free model structure. However, in practical applications with noisy data, the model appears to be practically non-identifiable in some cases. The problem is exacerbated when there is limited information content within the clinical data. To increase the model utility, more robust parameter identification is required and a requirement-profile for the data quality must be elaborated.

The proposed respiratory model incorporates the well known recruitment principle in a time-continuous model that is theoretically identifiable and accurately describes observed clinical dynamics of ARDS patients. Optimal ventilation management and continuous patient monitoring may profit from further investigations in applying this model in conjunction with others to evaluate and guide mechanical ventilation therapy.

\section{ACKNOWLEDGEMENT}

The authors express their gratitude to the McREM Study Group and Dräger Medical for providing the data for this retrospective study. This work was supported by the Deutsche Forschungsgemeinschaft DFG under Grant MO 579/1-2 PAR (Protective Artificial Respiration) and the German Academic Exchange Service (DAAD).

\section{REFERENCES}

Albaiceta, G. M., Taboada, F., Parra, D., Luyando, L. H., Calvo, J., Menendez, R. and Otero, J. (2004). Tomographic study of the inflection points of the pressure-volume curve in acute lung injury. Am J Respir Crit Care Med, volume 170, 1066-72.

Bellu, G., Saccomani, M. P., Audoly, S. and D'angio, L. (2007). DAISY: A new software tool to test global identifiability of biological and physiological systems. Comput. Methods Prog. Biomed., volume 88, 52-61.

Crotti, S., Mascheroni, D., Caironi, P., Pelosi, P., Ronzoni, G., Mondino, M., Marini, J. J. and Gattinoni, L. (2001). Recruitment and derecruitment during acute respiratory failure: a clinical study. Am J Respir Crit Care Med., volume 1, 131-40.

Docherty, P. D., Chase, J. G., Lotz, T. F. and Desaive, T. (2011). A graphical method for practical and informative identifiability analyses of physiological models: a case study of insulin kinetics and sensitivity. Biomed Eng Online, volume 10, 39.

Gattinoni, L., Caironi, P., Cressoni, M., Chiumello, D., Ranieri, V. M., Quintel, M., Russo, S., Patroniti, N., Cornejo, R. and Bugedo, G. (2006). Lung recruitment in patients with the acute respiratory distress syndrome. $N$ Engl J Med., volume 354, 1775-86.

Hickling, K. G. (1998). The pressure-volume curve is greatly modified by recruitment. A mathematical model of ARDS lungs. Am J Respir Crit Care Med., volume 158, 194-202.

Kretschmer, J., Wahl, A. and Moller, K. (2011). Dynamically generated models for medical decision support systems. Comput Biol Med, volume 41, 899-907.

Kretschmer, J., Wahl, A. and Möller, K. (2010). On the Integration of Different Physiological Model Systems. In: 4th International Conference on Bioinformatics and Biomedical Engineering (iCBBE), 2010 Chengdu, China. IEEE.

Lozano, S., Möller, K., Brendle, A., Gottlieb, D., Schumann, S., Stahl, C. A. and Guttmann, J. (2008). AUTOPILOTBT: a system for knowledge and model based mechanical ventilation. Technol Health Care., volume 16, 1-11. 
Markhorst, D. G., Van Genderingen, H. R. and Van Vught, A. J. (2004). Static pressure-volume curve characteristics are moderate estimators of optimal airway pressures in a mathematical model of (primary/pulmonary) acute respiratory distress syndrome. Intensive Care Med, volume 30, 2086-93.

Pulletz, S., Adler, A., Kott, M., Elke, G., Gawelczyk, B., Schadler, D., Zick, G., Weiler, N. and Frerichs, I. (2011). Regional lung opening and closing pressures in patients with acute lung injury. J Crit Care, volume (in press).

Raue, A., Kreutz, C., Maiwald, T., Bachmann, J., Schilling, M., Klingmuller, U. and Timmer, J. (2009). Structural and practical identifiability analysis of partially observed dynamical models by exploiting the profile likelihood. Bioinformatics, volume 25, 1923-9.

Salazar, E. and Knowles, J. H. (1964). An analysis of pressure-volume characteristics of the lungs. $J$ Appl Physiol., volume 19, 97-104.

Schiller, H. J., Steinberg, J., Halter, J., Mccann, U., Dasilva, M., Gatto, L. A., Carney, D. and Nieman, G. (2003). Alveolar inflation during generation of a quasi-static pressure/volume curve in the acutely injured lung. Crit Care Med, volume 31, 1126-33.

Schranz, C., Docherty, P. D., Chiew, Y. S., Chase, J. G. and Möller, K. (2012). A Time-Continuous Model of Respiratory Mechanics of ARDS Patients. In: World Congress 2012 Medical Physics and Biomedical Engineering, 2012 Beijing, China. Springer.

Schranz, C., Knöbel, C., Kretschmer, J., Zhao, Z. and Möller, K. (2011). Hierarchical Parameter Identification in Models of Respiratory Mechanics. IEEE Trans Biomed Eng., volume 58, 3234-41.

Stahl, C. A., Möller, K., Schumann, S., Kuhlen, R., Sydow, M., Putensen, C. and Guttmann, J. (2006). Dynamic versus static respiratory mechanics in acute lung injury and acute respiratory distress syndrome. Crit Care Med., volume 34, 2090-8.

Sundaresan, A. and Chase, J. G. (2011). Positive End Expiratory Pressure in patients with Acute Respiratory Distress Syndrome - The past, present and future. Biomed Signal Proces, volume to appear.

Sundaresan, A., Chase, J. G., Shaw, G. M., Chiew, Y. S. and Desaive, T. (2011). Model-based optimal PEEP in mechanically ventilated ARDS patients in the intensive care unit. Biomed Eng Online, volume 10, 64.

Sundaresan, A., Yuta, T., Hann, C. E., Chase, J. G. and Shaw, G. M. (2009). A Minimal Model of Lung Mechanics and Model based Markers for Optimizing Ventilator Treatment in ARDS Patients. Comput Methods Programs Biomed., volume 95, 166-80. 